\section{A CASE OF CERVICAL RIB}

Obliteration of the Arteries of the Right Arm: Gangrene of a Porition of Several Fingers : ExcISION OF THE RIB.

Bx T. E. GORDON, M.B., B.CH., F.R.C.S.I., Surgeon to the Adelaide Hospital.

THE subject of the present communication is a case of arterial thrombosis affecting the vessels of the right arm, and its chief interest lies, not in its effects, but in speculation as to its cause.

History. - The patient, a farm labourer, aged ${ }_{32}$, was in good health until May, r899. He has never had syphilis nor is he an alcoholic. His first symptom was the occurrence of attacks of pallor and numbness in the fingers of his right hand when he got up in the mornings. At first the little finger was alone affected, but he afterwards noticed it in all the fingers. He suffered from no pain. Early in July his right hand becam weak. The weakness passed off after a few days, but the attacks of anæmia and numbness, which had ceased during June, reappeared. Now came red, swollen, cold, and painful. The pain was worse at night and he used to sleep in a chair because he had thus less pain than when
lying down. The pulse at the wrist ceased to be palpable in probably the
latter part of July.

Anamnesis. - He was admitted to the Adelaide Hospital on August 22nd, 1899. His right hand was then slightly swollen but did not pit on pres sure. The lower third of the forearm and the hand were dusky red an deady cold. below the junction of the middle and lower thirds of the brachial artery. The artery at the elbow was felt as a cord. On one occasion pressure over the brachial artery caused severe and dasting pain referred to the fingers. The pulse frequency was mo. Strong and visible pulsation was observed in the subclavian artery. It was doubtful if the pulse in the upper part of the brachial artery was less than on the opposite side.

Possessed of these facts I looked up all the literature I could find bearing on the case. In the course of my search I found a paper by Mr. Bowlby in the Lanoet for 1888 . 'The writer here stated his belief that many of the cases of socalled obliterative arteritis were in reality examples of cervical rib. This paper came to me as a veritable lux in tenebris, and I took an early opportunity to re-examine my patient, when, much to my pleasure and surprise, I found a bony ridge running across the posterior triangle of the neck on the right side. On the left I was unable to detect anything to correspond, yet a skiagraph subsequently taken showed the presence of a cervical rib on both sides, the right rib being higher and more nearly horizontal than its fellow.

After a few days in hospital the tip of the index finger became gangrenous, and gangrene also commenced in the middle and ring fingers. There was neither motor nor sensory paralysis.

I removed the rib on August 27 th, 1899 . A complete paralysis of the arm followed upon the operation. In spite of this the condition of the hand improved; gangrene of the index and other fingers advanced a destroyed, but only the tips of the other affected fingers. He was allowed to walk about early in November. When the arm hung down its condition reverted to that at the time of admission; the tip of the little finger became slaty-blue coloured. He no longer suffered from pain, and the effects of the paralysis were slowly disappearing. If the arm were raised up and back the pulse disappeared from the brachial and axillary arteries. It did not return

Whats were made.

What was the cause of the arterial obliteration? The obliteration of the arteries and the cervical rib are related as cause and effect. The occurrence of the two conditions, both very rare, in the same individual and in such a position as to make it possible that they should be causally related makes it probable that they are, and this even if the present case were unique. But there are a considerable number of other similar cases. One would indeed expect that the reappearance of a cervical rib would be attended with some form of inconvenience or danger to the individual, for were this not so it is difficult to see why in the course of development this rib should have been suppressed, and that a cervical rib is an atavism is, I think, allowed by all recognised anatomical authorities.

Cases of cervical rib may conveniently be grouped under three headings: (1) Where no symptoms are produced, and this is the largest class; (2) where the rib gives rise to nervous symptoms only, such as wasting and loss of power, pain, and chilliness; (3) where the leading symptoms are circulatory. Amongst these, which are extremely rare, I have found records of the following:

x. Holmes Coote's case, 186x. ${ }^{2}$ Radial, ulnar, and brachial arteries obliterated, pain in course of nerves. Cervical rib excised with satisfactory result.

2. Alfred Poland's case, $1869 .{ }^{3}$ Early symptoms, pains and weakness of right arm, also numbness, coldness, and evidence of deficient nutrition in nails, which were ill-developed and desquamating. Pulsating tumour above clavicle $;$, no doubt of its aneurysmal nature. Absence of arterial pulsation to within a shor by digital compression.

3. Hilton's case, 1876.4 Gangrene of fourth and fifth fingers. Pressure above clavicle at once caused pain in little and adjoining fingers.

4. T. Smith's case. 5 Loss of pulse in arm and forearm, also impaired sensation and motion in forearm. Whole arm became useless.

5. Pearce Gould's case. 6 The author reported the case as an example of "obliterative arteritis," and denied to the "exostosis" (cervical rib) any share in its production. There was gangrene of several finger-tips. The further progress of this case, as noted aiter a lapse of two years, is full of present importance. Four months before he came under observation for the second time, "he suddenly lost power in the left arm, and the same evening he became unconscious. Next day he spoke and moved his arm." "The right superficial temporal artery cannotbe right common in front of the tragus, the left is very readily relt. The righ in the fifth left interspace vertically below the nipple."

6. Hadden's case. ? First symptoms, severe pain in right arm shooting down to hand. End of index. middle, and right fingers become gandrenous seen two years after the first observation. The skin over the two last phalanges of affected fingers described as red, smooth, and shining. Patient complained that fingers of right (that is, affected) hand became very cold in winter. In this case no cervical rib was found at first though carefully looked for. It was, however, eventually found, and was excised by Morrant Baker.

7. Walsham's case. ${ }^{8}$ This case resembles the others in most of its symptoms, but no cervical rib was found. It is possible that a skiagraph the close resemblance of ve symptoms to those presented by my patient and others where a cere Con writes. "F the cause of obliteration of the arteries it will be found to depend on some nerve lesion. Peripheral neuritis involving chiefly the nerves supplying the vessels.

We may then confidently affirm that the arterial occlusion in my patient was in some way the consequence of the presence of the cervical rib. This result was not produced by the rib pressing upon the subclavian artery so as to obstruct its lumen, for the pulse above the middle of the arm was quite as good as on the other side. Again, it was not due to embolism, for the onset was not sudden; nor will embolism explain the early symptoms. Although the course of the case was not acute it, was not extremely chronic, for it attained to full development within three months. Moreover, in cases where the advance of the arterial obliteration has been observed, as in Pearce Gould's case, such advance was fairly rapid. Thus, on November igth he found the brachial artery pulseless as high as the junction of its middle and upper thirds. One week later it was pulseless at the middle of the arm, and in one week more there was no pulse up to one inch below the axilla. From this it seems safe to conclude that the artery was obliterated by a thrombosis as opposed to occlusion by obliterative arteritis. Moreover, to suppose a spreading inflammation of the whole arterial tree of the upper limb in the absence of micro-organisms seems scarcely consistent with generally recognised pathological principles.

The spread of a thrombosis implies a preceding abnormal condition of the walls of the arteries affecting their endothelial lining. On this point it is necessary to draw special attention to the man's occupation. He was a farm labourer, and part of his work was to carry heavy buckets of food for cattle. Gould's patient was a labourer in a brick-field, Poland's was the same ; Walsham's patient did heavy manual labour ; Hadden's was a children's nurse.

In the majority of the cases it was the right arm that was affected. I feel fairly confident, then, that the occupation of the patient had to do with the causing of an abnormal state of the arterial wall. It is obvious, however, that carrying heavy weights or other use of the arm is of itself an insufficient cause. We may conclude that the artery was, to a more than normal extent, vulnerable to external influences, and we may further conclude from what has already been said, that the artery owed this excessive vulnerability to the presence of the cervical rib.

I have drawn attention to the fact that the brachial pulse. in its upper part was as strong as on the opposite side, and I drew the sufficiently obvious conclusion that the rib had not obstructed the subclavian artery, but there was another curious feature of the case. After the operation-some months after-I noticed that when the arm was abducted the pulse entirely disappeared below the subclavian, nor did it return when the arm was again brought to the side. But 
if the arm was drawn well forwards and moved about in a rotatory fashion pulsation became once more readily perceptible. I believe this phenomenon to be easily explained. At the operation I entirely removed the anterior end of the rib, but I left untouched a tuberosity on the first rib, with which the cervical rib articulated (see skiagraph). Now, when the arm was drawn out and back the subclavian was slung over this projection, and if the arm were gently lowered it remained on the wrong side of the tuberosity, and was so compressed. I think it most unlikely that the artery was held in this way before operation, but it seems probable that the pulse was occasionally arrested in certain positions of the arm. In another case of cervical rib which I have seen Dr. Wallace Beatty noticed that when the patient drew a deep breath and held it the pulse on the left side entirely ceased until the chest began to sink in expiration. In this case also (the patient is a girl about 18 years of age) there is on the left side a tuberosity on the first rib. But the subclavian artery is not prominent and, therefore, lies in all probability in front of the tuberosity

and behind the scalenus anticus. I imagine that the loss of pulse during a deep inspiration (in which the scalenes are contracted) is due to the pressure of the artery against the tuberosity by the scalenus anticus muscle. Whether - this be a correct explanation or not the interesting fact remains that at certain times, in both these cases, the circulation in the upper extremity was interrupted to a greater or less extent.

Can these facts be held to throw any light on the present case? I think we may safely answer in the negative. But if we can exclude occlusion of the subclavian artery either in a temporary or per-

manent fashion and if we reject a spreading arteritis as cause and also embolism, it seems to me we have exhausted every possible explanation of the case as far as the mutual relations of artery and rib are concerned.

Mr. Bowlby writes: "My own opinion is that in some of these cases the impaired nutrition of the limb, the blue and cold fingers and the sluggish circulation are dependent on pressure on the brachial plexus, or perhaps, as in $\mathrm{Mr}$. Walsham's case, on neuritis." These remarks bring us face to face with a truly difficult problem. Is it possible that in the case under consideration the obliteration of the arteries was due to some nervous lesion?

In the first instance it will, I think, be accepted as a correct statement that where an artery and a nerve are equally exposed to pressure the nerve is more likely to suffer than the blood vessel. Crutch palsy is an example of this. The lowest nerves of the brachial plexus are in as intimate relation to the anterior end of the cervical rib as the subclavian artery, and they are also intimately related to the rib at its vertebral end. One would expect then that where a cervical rib gave rise to symptoms these would more commonly be nervous than circulatory. A study of a number of such cases fully justifies this expectation. Disturbance of circulation is extremely rare; the usual symptoms are of purely nerve origin, for example, pain, numbness, weakness, and muscular wasting. All this serves to emphasise the importance of considering the subject from this fresh standpoint.

A nerve lesion is capable of causing a terminal gangrene independent of vascular disease. We may regard this as an established fact.

I might quote numerous cases bearing on this point, in which gangrene was the result of peripheral neuritis or some other disease of nerves or nerve centres, but I should not thus throw much additional light on the present case, for two reasons. First, because there is no reason to believe that in such cases any alteration occurred in the vesselsindeed in some instances it is distinctly stated that there were no such changes; and secondly, because in those cases where gangrene supervened there were also present quite unequivocal signs of the nervous derangement which led to the gangrene. There is, however, evidence that nerve injury or disease can cause important changes in the walls of blood vessels.

It has been shown that injury to the main nerve trunks of a limb (in expe riments on animals) is capable of inducing some degree of structural change in the arteries. Thus Fraenkel found, after division of the sciatic nerve, changes in the anterior tibial artery similar to those he had found associated with tabes, peripheral neuritis, and 'syringomyelia. He has described the con-

dition under the name "nervous angio-sclerosis"

In an interesting paper quite recently published by Weir Mitchell and Spiller ${ }^{10}$ on a case of erythro-melalgia, the authors quote an observation of Thoma. 'This pathologist found in a case of supraorbital neuralgia very decided arteriosclerosis in the temporal artery.

The case which formed the subject of Weir Mitchell's paper was a man, aged 62, suffering from intense pain in his great toe of the right foot: the toe was slightly ren but not swollen. No pulse could be detected by Dr. Morton or Dr. Mitchell in the right leg below the groin. The great toe was amputated and submitted to careful examination. There was found intense degeneration of the nerves, and in the vessels the media was found thickened and "the intima intensely proliferated, so much so that it projects into the lumen of the vessels in great masses of unequal size. and in some places nearly closes the lumen

In Friediänder's obliterative arteritis the early symptoms often present a remarkable similarity to those of Ravnaud's disease, and the group of symptums included under the term "intermittent claudication" are often observed prior to any distinctive evidence of arterial disease. Moreover, quite extensive nerve changes have been found in cases of this kind. ${ }^{11}$ In his article on this disease in Allbutt's System, Mott 
writes: "I have recently seen a case of symmetrical gangrene of the lower extremities in a middle-aged man suffering from alcoholic neuritis, the cause of the gangrene being arteritis and thrombosis." I cannot dwell any further on this question but I hope it will be conceded that it is possible that a nerve lesion should cause arterial disease.

I stated that there was a second difficulty in bringing my case into harmony with other cases of gangrene of nervous origin, inasmuch as the latter have presented other and unequivocal signs of the nerve lesion. In my case the symptoms of nervous disorder were scarcely unequivocal. There were, however, the temporary paresis of the hand, the pain referred to the fingers when pressure was made on the course of the brachial artery which, at the midarm, is also the course of the median nerve, and the intense pain which formed so prominent a feature of the case for some weeks before the gangrene commenced.

Now, although what I have said with regard to concomitant symptoms of nerve disease is very generally true, yet there are some cases recorded in which such symptoms were absent. In the paper just now referred to by $\mathrm{W}$. Mitchell, it is stated that all forms of sensation, tactile, thermic, and painful, were present in the toe, and yet the microscope showed a degeneration of a very marked character. The authors write, "Our case is not the cnly one in which the preservation of sensation has seemed almost inexplicable after a histological examination has been made revealing intense degeneration." This aspect of the subject leads me to draw attention to a matter of some importance. If my account of the case was in any way true to $\mathrm{Na}$ ture, no one will fail to have been struck by the general resemblance of the symptoms to the local phenomena of Raynaud's disease. There was the early ischæmia followed by dusky red suffusion and coldness, culminating in terminal gangrene. Now in Raynaud's disease we find a condition in which an effect is produced through some disorder of vasomotor nerves or centres, and in which there is frequently no evidence pointing to implication of other nerve elements, whether afferent or efferent.

But the analogy of such diseases as Raynaud's disease and erythomelalgia cannot be said to efface this difficulty that the pressure of the cervical rib should exhaust its evil influence on the vasomotor elements of a nerve trunk, and leave the other nerve fibres practically unscathed. Moreover, in Raynaud's disease it is generally stated that the arteries are perfectly normal structurally. This seems a fitting place to draw attention to some facts which, owing to Dr. Beatty's kindness, I had an opportunity of observing in the case of a young girl under his care at the Adelaide Hospital.
This patient had presented the classical signs of Raynaud's disease for a period of ten years. Now the hands, which had borne the chief brunt of the disease, were atrophied and the fingers tapering. On exposure to cold they assumed the characteristic livid coloration. The chief interest of the case, from our present point of view, lies in a fact observed by Dr. Beatty. He found that no pulse could be detected anywhere in the left arm, neither in the radial, nor brachial, nor even in the subclavian artery, whilst a pulse was easily discernible in the usual situations in the other arm.* It is obvious from this that extensive structural change had occurred in the arteries of the left arm. Some structural change has been noted by previous observers in the arteries in Raynaud's disease, but such marked change as had occurred here must, I imagine, be extremely rare. I wish further to draw special attention to the fact that there were present in this case none of the gross signs of a peripheral neuritis. Now, if we can regard Raynaud's disease in general, and this case in particular, as a pure vasomotor neurosis (and I think we can), then we may conclude that not only may arterial change result from injury or disease of nerves in general, but may also result from injury or disease of vasomotor nerves alone. In addition to a general reseniblance to Raynaud's disease, there are some other facts which, perhaps we may be allowed to think, point towards a sympathetic lesion.

In $\mathrm{my}$ case the pulse-rate was rio, and quite out of proportion to the slightly elevated temperature. Inordinate frequency of the pulse was noted as a remarkable feature in Gould's case, and after the symptoms

had persisted some time there

developed signs of hypertrophy of the heart.
Excessive pulsation in the subclavian is another not uncommon feature of these cases, and now and then an dilatation-" aneurysm"-has been observed. This was so in Poland's case and in Walsham's, and was associated with obliteration of the arteries of the arm. Walsham's patient presented this further peculiarity, that the size of the aneurysm varied from day to day, and even from hour to hour. One might suppose that this condition was due to local disease-atheroma of the subclavian artery-but this will not explain Walsham's case, nor will it explain the very common association of this dilatation with obliteration of the vessels of the arm (Fischer). I only know of one case where such an "aneurysm" occurring in conjunction with a cervical rib was submitted to dissection. This is Robert Adam's case. As the result of his investigation (post mortem), he found no evidence of any atheromatous disease of the wall of the aneurysm. ${ }^{12}$ Taking these facts into consideration, it * A very faint pulse was occasionally felt at the wrist, but it never approached in strength that of the opposite arm. 
seems to me probable that the dilatation may have been due in these various cases to loss of arterial tone.

One of the most carefully recorded cases of cervical rib that $I$ have come across is that of $M$. Planet. The patient here complained of nerve troubles only, pain, numbness, etc., and in particular of chilliness of the hand, on the side of the prominent cervical rib, on exposure to cold. There was nothing amiss with the circulation in the arm. This seems to me an important fact, and suggests a disturbance of the vasomotor mechanism. The man complained of some alteration of voice, and this was supposed to be due to some interference with the recurrent laryngeal nerve.

In Walsham's case there is another fact of singular interest. Whilst the patient-a man-was under observation, an enlargement of the lobe of the thyroid was observed on the same side as the dilated subclavian and obliterated arteries. In Dr. Beatty's case of Raynaud's disease there is also a thyroid enlargement (cystic in nature), and this association of goître and Raynaud's disease has been on several occasions noted by previous observers. Now I have no intention of entering into a discussion of the pathology of thyroid enlargements, but I can see nothing improbable in the supposition that such enlargements might occasionally result from some vasomotor fault. The results of the most recent operative treatment of exophthalmic goître are of interest in this connection. Jaboulay and others have excised a portion of the cervical sympathetic in such cases with beneficial effect, it is stated, not only on the exophthalmos, but also on the goître itself.

What I have endeavoured to show by all this is that there is some evidence in these cases pointing to a lesion of the sympathetic. It remains that I should suggest a possible explanation for the fact that these nerves should have suffered from the presence of the cervical rib. With this in view I made a careful dissection to demonstrate the normal relations and connections of the cervical sympathetic and the trunks of the brachial nerves. For the opportunity of making this dissection I am indebted to the kindness of Professor Cunningham. To avoid being wearisome I will consider together the normal anatomy and the alterations caused by the presence of the abnormal rib.

Normally, the eighth cervical and first dorsal nerves unite above the first rib, the last-named nerve rising to meet the other in intimate relationship with the neck of the rib. The neck of a cervical rib is crossed by the conjoined roots of the same nerves. and the seventh cervical nerve is a superior relation to the rib. I believe it is here-that is, at the neck of the cervical rib-that pressure symptoms are most likely to be produced. Since the cervical and first thoracic rib converge anteriorly, it is obvious that the displacement of normal relations will be greater the nearer the approach to the vertebral end. (It follows that the artery, which is only related to the cervical rib at its anterior end, will suffer relatively less displacement than the nerves.) The inferior cervical ganglion of the sympathetic dies normally in a recess occupied by loose fat between the head of the first rib and the transverse process of the seventh vertebra. In the presence of a cervical rib it will very probably be dislodged from this recess, and lie in the much less protected position in front of the head of the abnormal rib. The branches of the ganglion to the nerve trunks are most abundant as they concern the lower brachial nerves; that is, the nerves most endangered by the cervical rib. The branch to the seventh nerve normally passes through the foramen in the transverse process of the seventh vertebra. This connection must be completely altered in the presence of a cervical rib, but I have no knowledge of what its course actually is under such circumstances.

It is unfortunate that we do not possess more exact knowledge of the altered relations of the sympathetic; considerations, however, such as I have referred to are sufficient to show that the anatomy of the inferior cervical ganglion and its branches must undergo a very material derangement from the normal. Further, we may conclude that there is nothing unreasonable in supposing that the extra rib might produce its harmful effects rather at its vertebral than at its distal extremity; and, if this is so, it becomes easier to explain the singling out of vasomotor fibres than if the rib only acted at its anterior end.
For clearness sake I would briefly epitomise what has gone before as follows :

I. The cervical rib was in some way the cause of the arterial obliteration.

2. The circulation through the subclavian was not obstructed.

3. The occurrence of embolism may be excluded.

4. The manner of the obliteration suggests thrombosis, which in turn implies a preceding disease of the endothelium.

5. The cause of this arterial disease was very probably in part, but only in part, the carrying of heavy weights.

6. If the rib acted directly on the artery, it must have done so in some way upon which present pathology throws no light.

7. The rib may have acted indirectly on the artery through the nerves.

8. The nerves are more likely to have suffered than the artery and nervous symptoms are much more common than circulatory.

9. A nerve lesion is capable of causing gangrene.

10. A nerve lesion may cause structural changes in the blood vessels.

II. The absence of clear signs of a peripheral neuritis is a striking argument against the theory of nerve origin.

12. My case resembles the local phenomena of Raynaud's disease, which is almost certainly a neurosis, and here there is commonly an absence of usual symptoms of neuritis.

13. But in Raynaud's disease the arteries are stated to be usually healthy. In Dr. Beatty's case, however, and in some others. this is certainly not so.

14. From the occurrence of such changes in Raynaud's disease we may conclude that not only from nerve lesions generally, but from disease of vasomotor nerves in particular, structural changes in arteries may arise.

15. In cases of cervical rib there occasionally occur symptoms which may possibly be explained by injury to the cervical sympathetic. Such are (I) increased heart action ; (2) excessive subclavian pulsation, and sometimes fusiform dilatation of the artery; (3) abnormal sensitiveness of the arm affected to external cold ; (4) thyroid enlargement.

16. A study of the anatomy of the region concerned suggests that the abnormal rib may have excited its evil effects not at its distal but at its proximal extremity; here the rib is an intimate relation not only of the seventh and eighth cervical and first dorsal nerves, but also of the inferior cervical sympathetic ganglion and its branches. The number of these sympathetic connections is much greater with the lower than the upper brachial nerve trunks.

\section{REFERENCES}

1 Wiedersheim, The Structure of Man, p. 36. 2 Med. Times and Gaz., 186I, vol. î, p. ro8. 3 Med. Chir. Trans., vol. lii, p. $275 .{ }^{4}$ Rest and Pain, p. 200. St. Bartholomew's Hospital Reports, vol. Xxiv, p. 290. a Clin. Soc. Trans. vol. xvil, p. 95. 1i Dutil et Lamy, Arch. de Méd. Expér., vol. $\mathbf{1}$, 1893. ${ }^{12}$ For report of case see Poland's paper.

\section{THE EFFECT OF THE ROENTGEN RAYS IN A CASE OF CHRONIC CARCINOMA OF THE BREAST.}

BY ANDREW CLARK, F.R.C.S., Surgeon to the Middlesex Hospital and Lecturer on Surgery in the Medical School

THE following case seems worthy of putting on record seeing the remarkable improvement which has taken place since the regular employment of the Roentgen rays, now some nine weeks. A.B., a woman aged 60 , was admitted into the cancer Department of the Middlesex Hospital under my care on September igth, 1898, and the following is the note of her condition taken the next day:

The right breast consists almost entirely of a red ulcerating surface with a hard margin, the whole is fixed firmly to the pectoral muscle and bony structures beneath, and under the skin at the edge of the ulceration, towards the axilla a hard fixed mass can be felt. In the axilla several indurated glands can be distinguished, they are about the size of a large hazel nut and immobile. No other enlarged glands can be felt and the other breast appears normal. With the exception of several punched-out deep ulcers on both legs the patient apparently is in good health. 